\title{
Datos normativos para el Test de los Cinco Dígitos: desarrollo evolutivo de la flexibilidad en Educación Primaria
}

\author{
Cristina Rodríguez, Juan E. Jiménez, Alicia Díaz, \\ Eduardo García, Raquel Martín y Sergio Hernández \\ Universidad de La Laguna (España)
}

\begin{abstract}
El objetivo de este trabajo ha sido evaluar el desarrollo evolutivo de flexibilidad cognitiva y obtener datos normativos de la prueba "Test de los Cinco Dígitos" en escolares de Educación Primaria (EP) en la Comunidad Autónoma de Canarias. Con este propósito se seleccionó una muestra de 1.032 alumnos escolarizados entre primero y sexto curso de EP, con edades comprendidas entre los 6 y 12 años. Se analizó el tiempo invertido en la realización de las tareas de lectura, conteo y alternancia y calculándose finalmente el de flexibilidad. Los resultados encontrados ponen de manifiesto una tendencia lineal en el desarrollo de esta función, produciéndose un mayor crecimiento entre los 6 y 9 años y un descenso en la tasa de crecimiento entre los 9 y 13 años. Se calcularon también los baremos por curso de la prueba en la población escolar seleccionada de la Comunidad Autónoma de Canarias.
\end{abstract}

Palabras clave: Coste del cambio, evaluación, flexibilidad, funciones ejecutivas, Test de los Cinco Dígitos.

Normative data for the Five Digit Test: development of evolutionary flexibility in Elementary Education. The aim of this study was to analyze the development of flexibility and obtain normative data of the "Test of the five digits" in Primary school (EP) in the Canary Islands. For this purpose, a sample of 1.032 students was selected 1st to 6th grade belonging to school in Canary Islands with and age range between 6 and 12 years. The results show a linear trend in the development of flexibility. There was a significant increase in growth between 6 and 9 years and a decrease in growth rate between 9 and 13 . Normative data were also calculated by FDT test in the school population of the Canary Islands.

Key words: Flexibility, switch cost, task switching, assessment, executive functions, Five Digit Test.

Correspondencia: Cristina Rodríguez. Departamento de Psicología Evolutiva y de la Educación. Universidad de La Laguna. Campus de Guajara, C.P. 38205, La Laguna, Tenerife (España). Email. crodri@ull.es 
La flexibilidad es una habilidad cognitiva que se enmarca dentro del constructo psicológico denominado funciones ejecutivas (FE). Las FE han sido definidas de una gran diversidad de formas, y Barroso, Martín y León Carrión (2002) las definen como un conjunto de capacidades que permiten la generación de las diferentes acciones cognitivas necesarias para funcionar de forma organizada, flexible y eficaz, facilitando la adaptación del individuo a las diversas situaciones nuevas que le acontecen. Con respecto al núcleo de este estudio y de acuerdo con Anderson (2002), la flexibilidad cognitiva es la habilidad para cambiar o alternar un conjunto de respuestas, aprender de los errores, utilizar estrategias alternativas, dividir la atención y procesar múltiples fuentes de información simultáneamente.

La relación entre las diferentes funciones ejecutivas como entidades independientes o como parte de un todo ha sido objeto de debate y ha provocado la realización de diferentes estudios (v.gr. Isquith,Crawford, Espy, y Gioia, 2004; Miyake et al., 2000). Una de las teorías que trata de dar respuesta a esta disyuntiva es la teoría elaborada por Miyake et al. (2000) que pone de manifiesto la posibilidad de que a pesar de que creen una unidad que mide un constructo común, cada una de ellas hace aportaciones individuales a ese constructo. A partir de un análisis factorial confirmatorio utilizando como variables latentes inhibición, memoria de trabajo y "shifting”, encuentra que cada una de ellas aporta explicación diferenciada al constructo de las FE. Estos resultados se extrajeron a partir del estudio de una muestra de adultos jóvenes, sin embargo, han sido replicados en muestras de niños de 8 a 13 años de edad (Lehto, Juujärvi, Kooistra y Pulkkinen, 2003). En el caso de los niños se aboga por una independencia total entre las diferentes dimensiones (Hughes, 1998). Otros estudios en esta misma línea han encontrado sólo dos factores: memoria de trabajo y flexibilidad (v.gr. Huizinga, Dolan y Van der Molen, 2006).

En general, se ha utilizado el paradigma de la tarea de cambio o "task switching" para evaluar o medir el rendimiento en flexibilidad cognitiva (Allport et al., 1994; Rogers y Monsell, 1995) tomándose como variables tiempos de reacción, aciertos y errores perseverativos. En una tarea que requiere flexibilidad, la atención se desplaza de un set de estímulos a otro set y es el sistema de control quien debe permitir simultanear entre dos sets diferentes (Stuss, Floden, Alexander, Levine y Katz, 2001). En estas tareas se produce un efecto denominado el coste por el cambio de tarea o "swith cost", que indica la mayor lentitud o bien, menor exactitud en la respuesta de los sujetos cuando tienen que ejecutar un cambio en el criterio de elección o respuesta, cuando previamente habían estado utilizando otro, en comparación con el rendimiento conseguido cuando ejecutaban los ítems en los que no se había producido un cambio de criterio.

Es común encontrar instrumentos en los que se pueda medir tanto flexibilidad cognitiva como inhibición. Esto se debe, en parte, a que las demandas necesarias para la 
realización de las tareas en cada caso pueden estar relacionadas. En ambas tareas se deben gestionar una serie de recursos determinados que le permitan inhibir la respuesta más automática, sin embargo se trata de dos procesos diferenciados. En las tareas que evalúan la flexibilidad, la demanda del individuo es alternar su atención de un aspecto a otro, según un criterio dado. Para poder acometer este objetivo el sistema de control debe inhibir selectiva y conscientemente una de las respuestas durante el cambio de criterio. En el caso de la inhibición la atención se mantiene fija en un tipo de estímulo, pero la aparición de interferencias tiende a provocar una respuesta automática que el sistema debe inhibir (Slachevsky et al., 2005).

Los estudios de pacientes con daño cerebral y también los estudios llevados a cabo con neuroimagen en sujetos normales han permitido detectar que es el córtex prefrontal la región del cerebro más relacionada con las FE y, por lo tanto, con la flexibilidad. A pesar que desde temprana edad se pueden observar algunas de las funciones ejecutivas, hay que esperar hasta la adolescencia para observar un nivel de rendimiento similar a la de un adulto (Romine y Reynolds, 2005). Estos acontecimientos guardan una estrecha relación con los procesos madurativos que tiene lugar en la corteza cerebral en estas edades. Por tanto, es esperable encontrar patrones de activación variables en función de la edad. Rubia et al. (2006) encontraron durante una tarea de cambio una mayor activación en el córtex frontal inferior, en la región parietal y en la circunvolución cingular anterior, hallándose por otro lado, una disminución en la región dorsolateral durante la adolescencia. Más concretamente, algunos estudios de neuroimagen hacen referencia a la localización de esta habilidad en la zona media de la región dorsolateral (v.gr. Crone, Wendelken, Donohue y Bunge, 2006). En una revisión llevada a cabo por Shallice, Stuss, Picton, Alexander y Gillimgham (2008), se pone de manifiesto que a pesar de la divergencia en los resultados de los estudios llevados a cabo con neuroimagen con el objeto de poder describir con mayor precisión las áreas relacionadas con las tareas de cambio, los estudios y meta-análisis revisados coinciden, según los autores, en la implicación del córtex lateral prefrontal izquierdo. Aunque por otro lado, se constatan diferencias en regiones específicas. Se llama también la atención sobre la importancia, de las tareas utilizadas, así como de las variables medidas como fuente de discrepancias.

En el caso de los niños con trastorno por déficit de atención con/sin hiperactividad (TDAH), Etchaperaborda y Mulas (2004) encontraron que el 38\% tienen afectada la capacidad de flexibilidad cognitiva, y sugieren que quizás este grupo podría corresponder a un grupo más complejo que no responde con tanto éxito a los estimulantes. En este sentido, Etchaperaborda et al. (2004) proponen buscar patrones de activaciones diferentes en función del subtipo que presentan los niños con TDAH. Para ello seleccionaron diferentes subgrupos de niños con perfil de inatento y combinado y también un grupo control. Encontraron que el grupo combinado cometía un mayor 
número de perseveraciones en comparación al grupo control. Con respecto al patrón de activación, en el único grupo en que se encontró una mayor activación entre la condición de cambio frente a la de no cambio, fue en el grupo control, frente a los dos grupos con TDAH. La activación del grupo control fue encontrada en el cingulado anterior del hemisferio izquierdo inmediatamente después de recibir el feedback para cambiar de condición. Tras esta retroalimentación la activación presentada por el grupo control fue mayor que la del grupo combinado. Otra área en la que se apreciaron diferencias entre el grupo control y el resto de los grupos, fue en la corteza prefrontal dorsolateral del hemisferio izquierdo. Sin embargo, esta zona se activó en ambas condiciones lo que implica, que la participación de esta región no es específica de esta función.

En resumen, como se puede apreciar hay una gran divergencia en los resultados, debido a las diferencias principalmente metodológicas que se dan en los diferentes trabajos: tipos de sujetos seleccionados, instrumentos utilizados, edades de las muestras, etc.

Con respecto al desarrollo evolutivo, los estudios llevados a cabo siguiendo el paradigma de "task switching" han comprobado que la flexibilidad cognitiva se desarrolla y mejora con la edad (v.gr. Anderson, 2002; Garon, Bryson y Smith, 2008). En general se ha constatado que los niños a los 3 años de edad tienen un bajo rendimiento en tareas que requieran manejar más de un criterio, y que no son capaces de operar con dos pares de reglas incompatibles hasta los 5 años (García, Enseñat, Tirapu y Roig, 2009). Se demuestra también que se producen mejoras significativas entre los 7 y 15 años de edad (Huizinga, Dolan y Van der Molen, 2006). En esta línea, Davidson, Amso, Cruess y Diamond (2006) encuentran un progreso desde los 4 años hasta la adolescencia. Sin embargo estos resultados están mediatizados por el tipo de variable medida y también por las demandas de memoria de trabajo y de inhibición de las tareas propuestas. Los resultados mostraron que mientras el switch cost para la exactitud disminuye entre los 9 y 13 años, para los tiempos de reacción, se incrementa desde los 6 años hasta la adultez temprana. Este efecto es justificado por los autores apelando a la capacidad metacognitiva de los sujetos, esto es, a medida que los sujetos se hacen mayores modulan la velocidad de la respuesta para asegurar la exactitud de la misma. A pesar de que estos hallazgos han sido replicados en otros estudios existen otras investigaciones que han encontrado resultados opuestos. Reimer y Maylor (2005) encontraron que el switch cost para la variable tiempo sufría un descenso lineal entre los 10 y 18 años de edad. Los resultados de los estudios que utilizan muestras de sujetos adultos también son contradictorios.

Existen diferentes patologías infantiles que cursan con un déficit en flexibilidad cognitiva. Dentro de la categoría diagnóstica de los trastornos del desarrollo, destacamos el autismo como una patología caracterizada, entre otras cosas, por mostrar un déficit en funciones ejecutivas, principalmente déficit en el razonamiento verbal, 
memoria verbal y la flexibilidad cognitiva (v.gr. Liss, Fein, Alleng, Dunn y Feinstein, 2001; Ozonoff, Pennington y Rogers, 1991). El TDAH es otra de las patologías más estudiadas en torno al déficit en las funciones ejecutivas y más concretamente a la flexibilidad cognitiva. En general la mayor parte de los estudios llevados a cabo coinciden en el hecho de que los niños que padecen este trastorno suelen cometer un mayor número de errores perseverativos en comparación a los grupos controles (v.gr. López, Gómez, Aguirre, Puerta y Pineda, 2005; Máximo, Simonini y Delgado, 2004).

Otro tipo de enfermedades mentales más frecuentes en edad adulta que se caracterizan por presentar un déficit en rigidez cognitiva es la esquizofrenia. Presentan dificultades para la realización de las tareas y comenten un gran número de errores de perseveración (Querejeta, Godoy y Mías, 2011; Semkovska, 2004), así como algunas dificultades que se asocian a la tercera edad (Lineweaver, Bondi, Thomas y Salmon, 1999).

Como hemos podido constatar hasta el momento la flexibilidad cognitiva dentro del conjunto de las $\mathrm{FE}$ es una dimensión especialmente importante $\mathrm{y}$ específicamente a la hora de estudiar el perfil cognitivo de los niños con TDAH. Por otro lado, hemos apostado por estudiar el desarrollo evolutivo de la flexibilidad cognitiva comparando año a año los grupos seleccionados, puesto que este nivel de detalle puede facilitar la labor de los profesionales. Por tanto, el objetivo que persigue este estudio es el de explorar el rendimiento en flexibilidad cognitiva en una muestra de niños en edad escolar obteniendo las puntuaciones normativas para la población escolar canaria. Así como el estudiar el desarrollo de la habilidad a lo largo de la Educación Primaria, utilizando para ello un diseño transversal.

\section{MÉTODO}

\section{Participantes}

La muestra de estudio estuvo formada por un total de 1.032 niños canarios escolarizados en colegios públicos en la Etapa de Educación Primaria, de edades comprendidas entre los 6 años y 3 meses y los 12 años y 4 meses. Los centros seleccionados estaban localizados en zonas del centro y de la periferia del municipio de Santa Cruz de Tenerife. Debido a la pérdida de algunos datos por parte de 3 sujetos, los mismos fueron eliminados de este estudio quedando la muestra final formado por 1.029 niños. La distribución de la muestra por edad y sexo se presenta en la Tabla 1. No hubo diferencias significativas en la distribución del sexo en función del curso $\chi^{2}(5)=7.56$; $p=.182$.

\section{Instrumento}

Test de los Cinco Dígitos (Sedó, 2007). El objetivo de esta prueba es, tal y como lo manifiesta el propio autor, describir la velocidad y la eficiencia del procesamiento cognitivo, la atención sostenida, la automatización y la capacidad para 
gestionar y modular el propio esfuerzo mental cuando las series van aumentando su dificultad. Las diferentes condiciones que veremos a continuación permiten discriminar dificultades neurológicas a partir de la desaceleración de los sujetos durante la realización de las tareas propuestas. Este instrumento surge, con el objeto de eliminar ciertas limitaciones encontradas en el test de Stroop, como no poder administrarse a prelectores o bien a sujetos con problemas de lectura y tampoco a personas con problemas de percepción.

Tabla 1. Distribución de la muestra en función de la edad y el sexo

\begin{tabular}{|c|c|c|c|c|}
\hline & & \multicolumn{2}{|c|}{ SEXO } & \multirow{2}{*}{ Total } \\
\hline & & Varón & Mujer & \\
\hline \multirow{7}{*}{ EDAD } & Grupo 1 & 100 & 102 & 202 \\
\hline & Grupo 2 & 87 & 80 & 167 \\
\hline & Grupo 3 & 90 & 89 & 179 \\
\hline & Grupo 4 & 84 & 71 & 155 \\
\hline & Grupo 5 & 66 & 98 & 164 \\
\hline & Grupo 6 & 82 & 80 & 162 \\
\hline & Total & 509 & 520 & 1029 \\
\hline
\end{tabular}

El test presenta cuatro condiciones diferentes que se secuencian en orden creciente de dificultad. En cada una de estas situaciones se le presenta al individuo una lámina con 50 estímulos distribuidos en cinco columnas con diez filas cada una. Los estímulos son agrupaciones de asteriscos o dígitos que se muestran encuadrados en pequeños rectángulos. Las situaciones de evaluación son cuatro: lectura, conteo, elección y alternancia. Las dos primeras requieren un procesamiento cognitivo automático de los estímulos, puesto que se trata, en el primer caso, de leer el número que se repite, y en el segundo, en contar los asteriscos que aparecen delimitados en el rectángulo. Las cantidades y los dígitos que aparecen no superan el 5. Sin embargo el nivel de dificultad aumenta en las dos condiciones restantes denominadas, "elección" y "alternancia". En esta segunda parte del test, se presentan los dígitos en cantidades distintas a la que indica el valor numeral del propio dígito. Este tipo de presentación obliga al individuo a realizar operaciones conscientes y de control para seleccionar la respuesta correcta, puesto que difieren si se trata de contar o leer. En otras palabras, en la situación de "elección" el sujeto debe contar la cantidad de dígitos que aparecen en el recuadro, sin embargo la disposición espacial en la que se presentan le genera un conflicto, ya que hace que el individuo tenga una tendencia automática a leer el número y no a contarlos como se espera. Finalmente en la condición de "alternancia", los sujetos deben "alternar" ambos criterios, es decir deben contar en el $80 \%$ de los casos y cambiar de criterio y leer en el $20 \%$ restante. Los sujetos deben cambiar del criterio principal, el de contar, al criterio secundario, el de leer, siempre que se encuentren con un rectángulo cuyo marco tenga un grosor superior al habitual. Este test permite obtener dos puntuaciones más a partir de las puntuaciones directas obtenidas, que son las puntuación de la inhibición de las respuestas (Inhibición $=$ Elección-Lectura) $[$ Los datos referentes a esta dimensión se 
muestran en el estudio que se presenta en este monográfico bajo el título: "Datos normativos para el Test de Stroop: patrón de desarrollo de la inhibición y formas alternativas para su evaluación"] y la flexibilidad mental $($ Flexibilidad = AlternanciaLectura). Las características psicométricas de este test han sido probadas con muestras de diferentes países, entre ellas una muestra de adultos españoles. Los coeficientes de fiabilidad obtenidos a partir del procedimiento de las dos mitades y utilizando los índices de Spearman-Brown oscilaban en las cuatro tareas entre .86 a .94 en la muestra de adultos normales, y entre .89 y .92 para adultos con accidentes cerebro-vasculares. La validez del instrumento, fue llevada a cabo originalmente en una muestra con sujetos canadienses, concretamente 40 sujetos con problemas neuropsicológicos, encontrándose correlaciones significativas entre este instrumento y otros que también medían alternancia (LAT, Letter Alternation Test y Trial Making Test, TMT) (McLachlan y Sedó, 2003a). Sedó y DeCristoforo (2001) encuentran una correlación significativa de .65 a .71 entre las diferentes secciones del FDT y el test de Stroop. En cuanto al efecto de las variables de la edad y el sexo en el rendimiento de este test, los resultados varían en función de los estudios. Así en el estudio llevado a cabo por Walker (2003) (con sujetos clínicos) no se encuentra relación alguna entre la edad y el rendimiento en FDT, sin embargo en los estudios llevados a cabo con muestras españolas (con sujetos normales) sí se encuentra relación entre las variables obteniéndose una correlación negativa en niños y adolescentes (a mayor edad menor tiempo invertido) y positiva en adultos (a mayor edad más tiempo invertido). En cuanto al sexo, no se encuentran diferencias significativas entre hombres y mujeres ni en la muestra de niños ni en la de adultos, aunque en ambos casos las mujeres parecen invertir menos tiempo en la ejecución de la prueba.

\section{RESULTADOS}

Para poder acometer el primer objetivo de describir el desarrollo evolutivo del rendimiento en flexibilidad de los niños, se llevó a cabo, en primer lugar, un ANOVA con variable independiente "EDAD", con seis niveles: Grupo 1 (6 años y 3 meses - 7 años y 4 meses); Grupo 2: (7 años y 5 meses -8 años y 4 meses); Grupo 3 (8 años y 5 meses - 9 años y 4 meses); Grupo 4 (9 años y 5 meses -10 años y 4 meses); Grupo 5 (10 años y 5 meses - 11 años y 4 meses); Grupo 6 (11 años y 5 meses - 12 años y 4 meses) y como variables dependientes, las dimensiones obtenidas en el test (lectura, conteo, alternancia y flexibilidad). Debido al incumplimiento del supuesto de homogeneidad de varianzas, describiremos los resultados a partir de la corrección de Welch. Los resultados obtenidos muestran diferencias significativas entre los diferentes niveles de la variable EDAD, para cada una de las variables dependientes: para lectura, $\left[F_{\text {asintótica }}(5,475.63)=108.88, p<.001, \omega_{\text {estimada }}=.34\right]$; conteo, $\left[F_{\text {asinótica }}(5,475.42)=113.42\right.$, 
$\left.p<.001, \omega_{\text {estimada }}=.35\right] ;$ alternancia, $\left[F_{\text {asintótica }}(5,474.90)=144.10, p<.001, \omega_{\text {estimada }}=.41\right] \mathrm{y}$ flexibilidad, $\left[F_{\text {asintótica }}(5,476.47)=92.09, p<.001, \omega_{\text {estimada }}=.31\right]$. Las comparaciones a posteriori de las diferencias par a par así como las medias y las desviaciones típicas de los grupos en cada tarea se muestran en la tabla 2.

Tabla 2. Medias y desviaciones típicas por grupos de edad en cada una de las subtareas, y valores de $t$.

\begin{tabular}{|c|c|c|c|c|c|c|c|}
\hline & GRUPOS & $\mathrm{M}(\mathrm{DT})$ & Grupo 2 & Grupo 3 & Grupo 4 & Grupo 5 & Grupo 6 \\
\hline \multirow{6}{*}{ 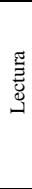 } & Grupo 1 & $36.68(9.81)$ & $t(367)=9.27 * * *$ & $t(379)=13.79 * * *$ & $t(355)=17.47 * * *$ & $t(364)=19.16^{* * * *}$ & $t(362)=22.38^{* * * *}$ \\
\hline & Grupo 2 & $30.59(6.07)$ & & $t(344)=4.15^{* * *}$ & $t(320)=8.03^{* * *}$ & $t(329)=9.50^{* * * *}$ & $t(327)=12.61 * * * *$ \\
\hline & Grupo 3 & $27.78(5.00)$ & & & $t(332)=4.09^{* *}$ & $t(341)=5.53^{* * *}$ & $t(339)=8.70^{* * * *}$ \\
\hline & Grupo 4 & 24.96 (4.67) & & & & $t(317)=1.33$ & $t(315)=4.40^{* * *}$ \\
\hline & Grupo 5 & $24.02(5.11)$ & & & & & $t(324)=3.12^{*}$ \\
\hline & Grupo 6 & $21.85(4.19)$ & & & & & \\
\hline \multirow{6}{*}{ ن. } & Grupo 1 & $50.71(15.46)$ & $t(367)=9.91 * * *$ & $t(379)=13.49 * * *$ & $t(355)=17.48 * * *$ & $t(364)=19.15^{* * *}$ & $t(362)=22.64^{*} * *$ \\
\hline & Grupo 2 & $40.89(9.64)$ & & $t(344)=3.24^{*}$ & $t(320)=7.44^{* * *} *$ & $t(329)=8.58^{* * *}$ & $t(327)=12.25^{* * *}$ \\
\hline & Grupo 3 & $37.59(8.07)$ & & & $t(332)=4.39 * * *$ & $t(341)=5.81^{* * *}$ & $t(339)=9.25^{* * * *}$ \\
\hline & Grupo 4 & $33.04(6.14)$ & & & & $t(317)=1.31$ & $t(315)=4.64 * * * *$ \\
\hline & Grupo 5 & $31.65(6.07)$ & & & & & $t(324)=3.38^{*}$ \\
\hline & Grupo 6 & $28.11(5.43)$ & & & & & \\
\hline \multirow{6}{*}{ 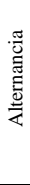 } & Grupo 1 & $101.89(26.22)$ & $t(367)=11.50^{* * * *}$ & $t(379)=17.47^{* * *}$ & $t(355)=20.91 * * *$ & $t(364)=23.23 * * *$ & $t(362)=26.10^{* * *}$ \\
\hline & Grupo 2 & $81.29(18.91)$ & & $t(344)=5.48^{* * *}$ & $t(320)=9.24 * * *$ & $t(329)=11.27 * * *$ & $t(327)=14.05^{* * * *}$ \\
\hline & Grupo 3 & 71.19 (13.92) & & & $t(332)=4.01 * *$ & $t(341)=6.00 * * *$ & $t(339)=8.85^{* * *}$ \\
\hline & Grupo 4 & $63.66(13.00)$ & & & & $t(317)=1.86$ & $t(315)=4.63 * * *$ \\
\hline & Grupo 5 & $60.09(11.54)$ & & & & & $t(324)=10.81$ \\
\hline & Grupo 6 & $54.76(11.16)$ & & & & & \\
\hline \multirow{6}{*}{ 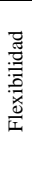 } & Grupo 1 & 64.74 (22.04) & $t(367)=9.02 * * *$ & $t(379)=14.42^{* * * *}$ & $t(355)=16.79 * * * *$ & $t(364)=19.03^{* * * *}$ & $t(362)=20.88^{* * * *}$ \\
\hline & Grupo 2 & $51.11(15.74)$ & & $t(344)=4.99 * * *$ & $t(320)=7.61 * * *$ & $t(329)=9.62 * * *$ & $t(327)=11.41^{*} * *$ \\
\hline & Grupo 3 & 43.34 (11.73) & & & $t(332)=2.85$ & $t(341)=4.81^{* * *}$ & $t(339)=6.66^{* * * *}$ \\
\hline & Grupo 4 & $38.83(10.90)$ & & & & $t(317)=1.86$ & $t(315)=3.64 * *$ \\
\hline & Grupo 5 & $35.82(9.86)$ & & & & & $t(324)=1.82$ \\
\hline & Grupo 6 & $32.91(10.06)$ & & & & & \\
\hline
\end{tabular}

Como podemos comprobar el patrón de diferencias entre los diferentes niveles de la variable edad fue similar para las variables de lectura, conteo y alternancia, es decir hubo diferencias entre todas las edades salvo en el rango comprendido entre los 9 y 10 años. Sin embargo en la puntuación de flexibilidad, calculada siguiendo las instrucciones del autor de la prueba (Flexibilidad= alternancia-flexibilidad), el patrón de resultados varía. Como se puede apreciar, inicialmente los dos grupos de edad más jóvenes se diferencian significativamente de todos los grupos, sin embargo las diferencias entre los tres grupos mayores no son tan grandes, encontrándose un patrón en el que las diferencias desaparecen entre los pares adyacentes. En otras palabras, parece haber un desarrollo más evidente en edades tempranas, sin embargo a partir de los 9 años y 3 meses se produce una desaceleración de esa evolución. A partir de los datos expuestos todo parece indicar que a medida que los niños van creciendo van mejorando en flexibilidad, en otras palabras, invierten menos tiempo para resolver la tarea. Sin embargo, debido a la desaceleración producida en los últimos grupos de edades, nos 
planteamos realizar un análisis de tendencia para poder describir con mayor precisión el patrón evolutivo de esta habilidad. Los datos resultantes indican que existe una relación lineal significativa entre la variable "EDAD" y la variable dependiente "Flexibilidad", $\left[F_{(5,1023)}=556,15, p<.001\right]$.

Figura 1. Desarrollo del rendimiento en flexibilidad medido en tiempo en función de la edad

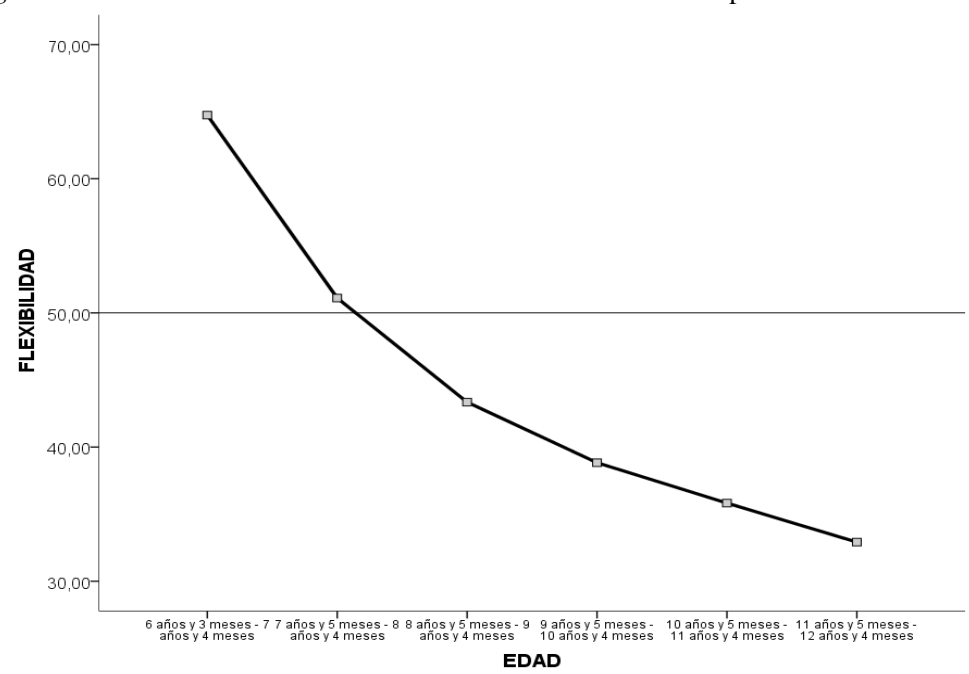

Tabla 3. Distribución de las puntuaciones en función de los cuartiles

\begin{tabular}{|c|c|c|c|c|c|}
\hline \multirow{2}{*}{ Grupo de Edad } & \multirow{2}{*}{ Percentil } & \multicolumn{4}{|c|}{ Medidas de tiempo en segundos } \\
\hline & & Lectura & Conteo & Alternancia & Flexibilidad \\
\hline \multirow{3}{*}{ Grupo 1} & 25 & 30.00 & 41.00 & 86.00 & 52.00 \\
\hline & 50 & 35.00 & 47.00 & 96.00 & 62.00 \\
\hline & 75 & 41.00 & 56.00 & 113.00 & 75.00 \\
\hline \multirow{3}{*}{ Grupo 2} & 25 & 27.00 & 34.00 & 68.00 & 41.00 \\
\hline & 50 & 30.00 & 40.00 & 79.00 & 48.00 \\
\hline & 75 & 34.00 & 45.00 & 91.00 & 58.00 \\
\hline \multirow{3}{*}{ Grupo 3} & 25 & 25.00 & 32.00 & 61,00 & 35.00 \\
\hline & 50 & 27.00 & 37.00 & 68.00 & 42.00 \\
\hline & 75 & 31.00 & 42.00 & 78.00 & 50.00 \\
\hline \multirow{3}{*}{ Grupo 4} & 25 & 21.00 & 28.00 & 56.00 & 31.00 \\
\hline & 50 & 25.00 & 32.00 & 62.00 & 37.00 \\
\hline & 75 & 28.00 & 36.00 & 70.00 & 45.00 \\
\hline \multirow{3}{*}{ Grupo 5} & 25 & 21.00 & 28.00 & 52.00 & 29.00 \\
\hline & 50 & 24.00 & 31.00 & 58.00 & 34.00 \\
\hline & 75 & 27.00 & 34.00 & 67.00 & 42.00 \\
\hline \multirow{3}{*}{ Grupo 6} & 25 & 20.00 & 24.00 & 47.00 & 26.00 \\
\hline & 50 & 21.00 & 27.00 & 52.00 & 30.00 \\
\hline & 75 & 24.00 & 31.00 & 60.00 & 39.00 \\
\hline
\end{tabular}

Nota. Grupo 1=6.3-7.4; Grupo 2=7.5-8.4; Grupo 3=8.5-9.4; Grupo 4= 9.5-10.4; Grupo 5=10.5-11.4 Grupo 6=11.5-12.4. Nota. Los percentiles han sido extraídos de las puntuaciones directas de los tiempos invertidos por los sujetos en la realización de cada subtest. No se han invertido las escalas, por lo que deberá ser interpretado de manera que a mayor percentil, peor rendimiento, puesto que invierte mayor tiempo.

En función de los resultados expuestos hasta el momento, resulta procedente calcular los baremos por año. En la tabla 3 se muestran los cuartiles de la variable 
tiempo tomado directamente de los tiempos invertidos por los sujetos para realizar cada una de las tareas, y que son necesarias para poder evaluar la flexibilidad cognitiva.

\section{DISCUSIÓN}

El objetivo de este trabajo fue estudiar el desarrollo evolutivo de la flexibilidad cognitiva de los niños canarios, así como la obtención de datos normativos para cada grupo de edad. Con respecto al desarrollo evolutivo de esta función, es obvia la tendencia lineal de la misma, a pesar de la desaceleración que se produce en los últimos años de la escolaridad en Educación Primaria. En este sentido, se constata la importancia de poder contar con datos normativos por año, puesto que existen diferencias significativas entre los diferentes grupos de edad. Estos resultados en relación a una mejora creciente de esta habilidad durante la etapa escolar ya han sido constatados en otros estudios utilizando otros instrumentos para la medición de esta habilidad (v.gr. Davidson, Amso, Cruess y Diamond, 2006). Tal y como se expuso en la introducción, la evaluación de esta habilidad en niños con TDAH podría favorecer la discriminación del perfil cognitivo del sujeto, facilitando una respuesta adecuada al mismo, ya que como encuentran Etchaperaborda et al. (2004) los niños con TDAH con un perfil combinado tienen un peor rendimiento en flexibilidad cognitiva en comparación al grupo control.

\section{Agradecimientos}

Esta investigación ha sido financiada por la Agencia Canaria de Investigación, Innovación y Sociedad de la Información de la Consejería de Educación, Universidades y Sostenibilidad del Gobierno de Canarias, con ref. PI 2007/012 del que es IP el segundo autor.

\section{REFERENCIAS}

Allport, A., Styles, E. y Hsieh, S.L. (1994). Shifting intentional set: Exploring the dynamic control of tasks. En C. Umiltá y M. Moscovitch (Eds.), Attention and Performance (p. 15). Cambridge, MA: MIT Press.

Anderson, P. (2002). Assessment and Development of Executive Function (EF) During Childhood. Child Neuropsychology, 8(2), 71-82.

Barroso, J.M. y Martín y León, J. (2002). Funciones ejecutivas: control, planificación y organización del conocimiento. Revista de psicología general y aplicada: Revista de la Federación Española de Asociaciones de Psicología. 55(1), 27-44.

Crone, E.A., Wendelken, C., Donohue, S.E. y Bunge, S.A. (2005). Evidence for separable neural processes underlying flexible rule use. Cerebral Cortex, 16(4), 475-86. 
Davidson, A.C., Amso, D., Anderson, L.C. y Diamond, A. (2006). Development of cognitive control and executive function from 4 to 13 years: Evidence from manipulation of memory, inhibition and task switching. Neuropsychologia, 44, 2937-2078.

Etchaperaborda, M.C. y Mulas, F., (2004). Flexibilidad cognitiva, síntoma adicional del trastorno por déficit de atención con hiperactividad. ¿Elemento predictor terapéutico? Revista de Neurología, 38(1), 97-102.

Etchaperaborda, M.C., Mulas, F., Capilla-González, A, Fernández-González, S., Campo, P., Maestú, F., Fernández-Lucas, A. y Ortiz, T. (2004). Sustrato neurofuncional de la rigidez cognitiva en el trastorno por déficit de atención con hiperactividad: resultados preliminares. Revista de Neurología, 38(1), 145-148.

García, A., Enseñat, A., Tirapu, J. y Roig, T. (2009). Maduración de la corteza prefrontal y desarrollo de las funciones ejecutivas durante los primeros cinco años de vida. Revista de Neurología, 48(8), 435-440.

Garon, N., Bryson, S.E. y Smith, I.M. (2008). Executive function in preschoolers: A review using an integrative framework. Psychological Bulletin, 134(1), 31-60.

Hughes, C. (1998). Executive function in preschoolers: lings with theory of mind and verbal ability. British Journal of Developmental Psychology, 16, 233-253.

Huizinga, M., Dolan, C.V. y Van der Molen, M.V. (2006). Age-realated change in executive function: Developmental trends and a latent variable analysis. Neuropsychologia, 44, 2017, 2036.

Isquith, P.K., Crawford, J.S., Espy, K.A., y Gioia, G.A. (2004); Assessment of executive function in preschool-aged children. Mental Retardation and Developmental Disabilities Research Reviews, 11(3), 209-215.

Miyake, A., Freidman, N.P., Emerson, M.J., Witzki, A.H., Howerter, A. y Wager, T.D. (2000). The unity and diversity of executive functions and their contributions to complex "frontal lobe" tasks: A latent variable analysis. Cognitive Psychology, 41, 49-100.

Lehto, J.E., Juujärvi, P., Kooistra, L. y Pulkkinen, L. (2003), Dimensions of executive functioning: Evidence from children. British Journal of Developmental Psychology, 21(1), 59-80.

Lineweaver, T., Bondi, M., Thomas, R. y Salmon, D. (1999). A normative study of Nelson's (1976) Modified Version of the Wisconsin Card Sorting Test in Helthy Older Adults. The Clinical Neuropsychologist, 13(3), 328-347.

Liss, M., Fein, D., Alleng, D., Dunn, M. y Feinstein, C. (2001). Executive functioning in Highfunctioning children with autism. Journal Child Psychology Psychiatry, 42(2), 261-270.

López, G., Gómez, L., Aguirre, D., Puerta, I. y Pineda, D. (2005). Componentes de las pruebas de atención y función ejecutiva en niños con trastorno por déficit de atención/hiperactividad. Revista de Neurología, 40(6), 331-339.

Máximo, C., Simonini, E. y Delgado, F. (2004). Flexibilidad cognitiva, síntoma adicional del trastorno por déficit de atención con hiperactividad: ¿elemento predictor terapéutico? Revista de Neurología, 38, 97-102.

McLachlan, J.F.C. y Sedó, M. (2003a). The development of a cross-cultural task of alternation. The Clinical Neuropsychologist, 17(1), 102. 
Ozonoff, S., Pennington, B. y Rogers, S. (1991). Executive function deficits in High-Functioning Autistic Individuals: Relationship to Theory of Mind. Journal of Child Psychology and Psychiatry, 32, 1081-1105.

Querejeta, A.N., Godoy, J.C. y Mías C.D. (2011) Abstracción verbal y flexibilidad cognitiva en esquizofrenia. Revista de Neuropsicologías, Neuropsiquiatrías y Neurociencias, 11(2), 85101.

Reimers, S. y Maylor, W. (2005). Task switching across the life span: Effects of age in general and specific switch cost. Developmental Psychology, 41, 661-671.

Rogers, R.D. y Monsell, S. (1995). Costs of a predictable switch between simple cognitive tasks. Journal Experimental Psychology General, 124, 207-231.

Romine, C.B. y Reynolds, C.R. (2005). A model of the development of frontal lobe function: Findings from a meta-analysis. Applied Neuropsychology, 12, 423-443.

Rubia, K., Smith, A.B., Woolley, J., Nosarti, C., Heyman, I., Taylor, E., et al. (2006). Progressive increase of frontoestriatal brain activation from childhood to adulthood Durant eventrelated tasks of cognitive control. Human Brain Mapping, 27, 973-993.

Sedó, M. y DeCristoforo, L. (2001). All languages verbal tests free from linguistic barriers. Revista Española de Neuropsicología, 3, 63-68.

Sedó, M. (2007). Test de los Cinco Dígitos. Madrid: T.E.A. Ediciones.

Semkovska, M. (2004). Assessment of executive dysfunction during activities of daily living in schizophrenia. Schizophrenia Research; 69(2-3), 289-300.

Shallice, T., Stuss, D.T., Picton, T.W., Alexander, M.P. y Gillimgham, S. (2008). Mapping task switching in frontal cortex through neuropsychological group studies. Frontiers in Neurosciensce, 2(1), 79-85.

Slachevsky, A., Pérez, C., Silva, J., Orellana, G., Prenafeta, M., Alegría, P. y Peña, M. (2005). Córtex prefrontal y trastornos del comportamiento: Modelos explicativos y métodos de evaluación. Revista Chilena de Neuro-Psiquiatría, 43(2), 109-121.

Stuss, D.T., Floden, D., Alexander, M.P., Levine, B. y Katz, D. (2001). Stroop performance in focal lesion patients: Dissociation of processes and frontal lobe lesion location. Neuropsychologia, 39(8), 771-786.

Walker, W.L. (2003). Study of the Five Digit Test across cultures. San Francisco: Wright Institute (tesis doctoral inédita). Disponible en UMI Disertation Services.

Recibido: 30 de septiembre de 2011

Recepción Modificaciones: 20 de marzo de 2012

Aceptado: 22 de marzo de 2012 